\title{
Discrete-time Retrial Queue with Bernoulli Vacation, Preemptive Resume and Feedback Customers
}

\author{
Peishu Chen, Yongwu Zhou, Changwen Li* \\ School of Business Administration, South China University of Technology (China) \\ cps8@sina.com, syw 666@,botmail.com, *Corresponding author: cwli2008@163.com
}

Received: April 2015

Accepted: September 2015

\section{Abstract:}

Purpose: We consider a discrete-time retrial queue where the retrial time follows a general distribution, the server subject to Bernoulli vacation policy and the customer has preemptive resume priority, Bernoulli feedback strategy. The main purpose of this paper is to derive the generating functions of the stationary distribution of the system state, the orbit size and some important performance measures.

Design/methodology/approach: Using probability generating function technique, some valuable and interesting performance measures of the system are obtained. We also investigate two stochastic decomposition laws and present some numerical results.

Findings: We obtain the probability generating functions of the system state distribution as well as those of the orbit size and the system size distributions. We also obtain some analytical expressions for various performance measures such as idle and busy probabilities, mean orbit and system sizes.

Originality/value: The analysis of discrete-time retrial queues with Bernoulli vacation, preemptive resume and feedback customers is interesting and to the best of our knowledge, no other scientific journal paper has dealt with this question. This fact gives the reason why efforts should be taken to plug this gap.

Keywords: discrete-time queue, Bernoulli vacation, preemptive resume, Bernoulli feedback, general retrial time, stochastic decomposition 


\section{Introduction}

More and more scholars are interested in analysis of discrete time queues due to their applications in communication system and other related areas (Bruneel \& Kim, 1992; Takagi, 1993; Singh \& Sreenivas, 2014; Wei, Qin \& He, 2014). The analysis of discrete-time queues is very important and more suitable than their continuous-time counterparts for modeling telecommunication and computer systems, on account of these systems operate on a discretetime basis where events can only happen at regularly spaced epochs. More importantly, discrete-time models can be used to deduce the results for continuous-time models but not vice versa (Bruneel \& Kim, 1992).

Various classes of vacation mechanism have been discussed in the literature. Takagi (1993) investigated on discrete-time systems with vacations in his splendid monograph. Zhang and Tian (2001) presented a discrete queue with multiple adaptive vacations where the number of vacation is considered as random variable. Luo, Huang and Ding (2014) studied on the departure process of discrete-time queue with randomized vacations.

Researches on Bernoulli vacation policy are relatively less. Servi (1986) and Ramaswamy and Servi (1988) have introduced the M/G/1 Bernoulli schedule: If the queue is empty after a service completion, then the server begins a vacation period. If there are customers in the orbit after a service completion, then another service begins with specified probability $p$ or a vacation period begins with supplementary probability $q=1-p$. At the end of a vacation period, the server waits for service the next customer. In recent years, Wenhui (2005) study a continues-time retrial queue with Bernoulli vacation. Samanta (2009) analyzed a GI/Geo/1 queue with single Bernoulli vacation based on exhaustive service. Wang (2012) extended a continues-time retrial queue with Bernoulli vacation to the discrete-time Geo/G/1 retrial queues with Bernoulli vacation.

Queueing models with preemptive resume phenomenon are characterized by the fact that arriving customers have the right to interrupt the customers in service and begin their own service with LCFS discipline. It occurs in many situations in our real life such as dealing with emergency patient in hospital and treating to transmission of information systems. Kumar, Vijayakumar and Arivudainambi (2002) studied an M/G/1 retrial system with two-phase service and preemptive resume. Wu, Liu and Peng (2011) investigated a discrete-time Geo/G/1 retrial queue with preemptive resume and collisions. Wei et al. (2014) discussed Geo/G/1 retrial queue with preemptive resume and Bernoulli feedback.

Retrial queues are characterized by the feature that customers who find the server busy upon arrival are forced to leave the server temporarily and repeat their demand after a random time. A more realistic retrial queue with feedback phenomenon happens in many real world situations: for example, in communication networks where data transmissions need to be ensured error free within a certain probability, feedback scheme is used to request retransmission of packets that are lost or damaged in communication systems. Choi and 
Kulkarni (1992) have investigated M/G/1 retrial queue with feedback. Kumar, Vijayalakshmi, Krishnamoorthy and Basha (2010) have analyzed a single server retrial queue with linear retrial rate, collisions and feedback customers.

The analysis of discrete-time retrial queues with Bernoulli vacation, preemptive resume and feedback customers is interesting and no work in this direction is found in the queueing literature at present. This fact gives the reason why efforts should be taken to plug this gap. The remainder of the paper is organized as follows. In Section 2, we give the mathematical model description of the considered queueing system. In Section 3, we study the Markov chain, and derive the probability generating functions of the system state, the orbit size and the system size distribution. We also obtain several performance measures of the system. In Section 4, we find two different stochastic decomposition laws. Some numerical results to illustrate the impact of the Bernoulli vacation policy, preemptive resume and feedback on the performance of the system are considered in Section 5. Finally, we give a conclusion in Section 6.

\section{Model Description}

We take into account a discrete-time single server retrial queue where the time axis is divided into slots and the time axis is marked by $0,1, \ldots, m, \ldots$. We further assume that all queueing activities (arrivals, departures, retrials, vacations) occur at the slot boundaries. Since in discrete time systems, many events may occur at the same time, we must impose additional assumptions to ensure that our model follows the evolution of the underlying process. For mathematical clarity, we suppose that the departures, Bernoulli feedback and the end of the vacations occur in the interval $\left(\mathrm{m}^{-}, \mathrm{m}\right)$ in sequence, and the primary arrivals, the preemptive resume, the retrials, the beginning of a vacation occur in the interval $\left(m, m^{+}\right)$in sequence, that is, early arrival scheme (known also as departure first rule) is adopted in this paper.

New customers arrive from outside of the system according to a geometrical arrival process with rate $p$. We assume that there is no waiting space in front of the server, and therefore, if an arriving customer finds the server idle, he begins his service immediately. Otherwise, if the server is busy at the arrival epoch, the arriving customer either interrupts the customer in service to commence his own service with probability $\alpha$ or leaves the server and enters the orbit with probability $\bar{\alpha}=1-\alpha$. The interrupted customer enters into the orbit. The server will take a single vacation with probability $\eta$ once he completes a service process, or start a process of search in order to find the next customer to be served with probability $\bar{\eta}=1-\eta$. If the system is on vacation, the arriving customer leaves the service area and enters the orbit. After vacation, the server becomes free and waits for the next customer. It is called "Bernoulli vacation" policy in this paper. After service completion, the customer decides either to join the orbit for another service with probability $\theta$ or leaves the system with complementary probability $\theta=1-\theta$. The customers in the orbit retry in accordance with a first-come, first- 
serve discipline, that is, only the customer at the head of the orbit queue is permitted to access to the server. Successive inter-retrial times of any customer are governed by an arbitrary distribution $\left\{a_{i}\right\}_{i=0}^{+\infty}$ with generating function $A(x)=\sum_{i=0}^{+\infty} a_{i} x^{i}$. Service times are governed by probability distribution $\left\{s_{i}\right\}_{i=1}^{+\infty}$ with generating function $S(x)=\sum_{i=1}^{+\infty} s_{i} x^{i}$ and the $n=t h$ factorial moment $\beta_{1, n}$. The vacation times are independent and identically distributed with arbitrary distribution $\left\{v_{i}\right\}_{i=1}^{+\infty}$, generating function $V(x)=\sum_{i=1}^{+\infty} v_{i} x^{i}$ and the $n=t$ factorial moment $\beta_{2, n}$. Here we pointed out that various stochastic processes involved in the system are assumed to be independent of each other. To avoid trivial cases, we suppose $0<p<1,0<\alpha<1,0<\theta<1$, $0 \leq \eta \leq 1$. The corresponding transition rate diagram is shown in Figure 1 .

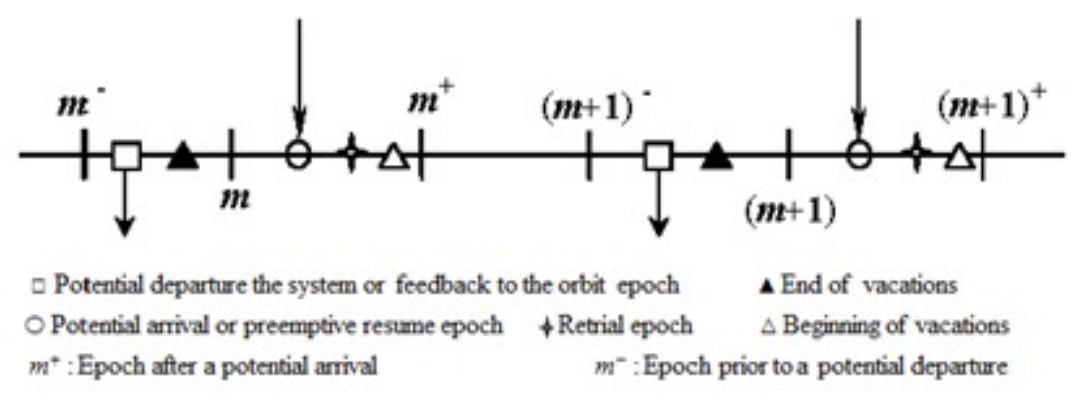

Figure 1. Various time epochs in an early arrival model

\section{Markov Chain}

At time $m^{+}$, the model can be described through the process $\left\{Y_{m}=\left(C_{m}, \xi_{0, m}, \zeta_{1, m}, \xi_{2, m}, N_{m}\right), m\right.$ $=0,1, \ldots\}$, where $C_{m}$ represents the state of the server; 0,1 or 2 according to whether the server is free, busy or on vacation, respectively; $N_{m}$ denotes the number of repeated customers in the orbit. If $C_{m}=0$, then $\zeta_{0, m}$ denotes the remaining retrial time. If $C_{m}=1$, then $\zeta_{1, m}$ denotes the remaining service time of the customer currently under service and if $C_{m}=2$, then $\xi_{2, m}$ corresponds to the rest vacation time. It can be proved that $\left\{Y_{m}, m \in N\right\}$ is a Markov chain with the state space $\{(0,0),(0, i, k): i \geq 1, k \geq 1 ;(1, i, k): i \geq 1, k \geq 0 ;(2, i, k): i \geq 1, k \geq 0\}$. In order to obtain the stationary distribution of the process, we define the stationary probabilities of the Markov chain $\left\{Y_{m}, m \in N\right\}$ as follows:

$$
\begin{aligned}
& \pi_{0,0}=\lim _{m \rightarrow \infty} P\left[C_{m}=0, N_{m}=0\right], \pi_{0, i, k}=\lim _{m \rightarrow \infty} P\left[C_{m}=0, \zeta_{0, m}=i, N_{m}=k\right], i \geq 1, k \geq 1, \\
& \pi_{1, i, k}=\lim _{m \rightarrow \infty} P\left[C_{m}=1, \zeta_{1, m}=i, N_{m}=k\right], i \geq 1, k \geq 0, \pi_{2, i, k}=\lim _{m \rightarrow \infty} P\left[C_{m}=2, \zeta_{2, m}=i, N_{m}=k\right], i \geq 1, k \geq 0,
\end{aligned}
$$


The Kolmogorov equations for the stationary distribution are given as follows:

$$
\begin{gathered}
\pi_{0,0}=\bar{p}\left(\pi_{0,0}+\bar{\theta} \bar{\eta} \pi_{1,1,0}+\pi_{2,1,0}\right) \\
\pi_{0, i, k}=\bar{p}\left(\pi_{0, i+1, k}+\theta \eta a_{i} \pi_{1,1, k-1}+\bar{\theta} \eta a_{i} \pi_{1,1, k}+a_{i} \pi_{2,1, k}\right), i \geq 1, k \geq 1, \\
\pi_{1, i, k}=\delta_{0, k} p s_{i} \pi_{0,0}+\left(1-\delta_{0, k}\right) \sum_{j=1}^{+\infty} \pi_{0, j, k} p s_{i}+\bar{p} s_{i} \pi_{0,1, k+1}+\left(1-\delta_{0, k}\right) p \theta \bar{\eta} s_{i} \pi_{1,1, k-1} \\
+\left(p \bar{\theta}+p \bar{\theta} a_{0}\right) \bar{\eta} s_{i} \pi_{1,1, k}+\bar{p} \bar{\theta} \bar{\eta} a_{0} s_{i} \pi_{1,1, k+1}+\left(1-\delta_{0, k}\right) p \bar{\alpha} \pi_{1, i+1, k-1}+\bar{p} \pi_{1, i+1, k} \\
+\left(1-\delta_{0, k}\right) p \alpha s_{i} \sum_{j=2}^{+\infty} \pi_{1, j, k-1}+p s_{i} \pi_{2,1, k}+\bar{p} a_{0} s_{i} \pi_{2,1, k+1}, i \geq 1, k \geq 0, \\
\pi_{2, i, k}=\bar{p} \bar{\theta} \eta v_{i} \pi_{1,1, k}+\left(1-\delta_{0, k}\right) p \bar{\theta} \eta v_{i} \pi_{1,1, k-1}+\left(1-\delta_{0, k}\right) \bar{p} \theta \eta v_{i} \pi_{1,1, k-1} \\
+\left(1-\delta_{0, k}\right)\left(1-\delta_{1, k}\right) p \theta \eta v_{i} \pi_{1,1, k-2}+\bar{p} \pi_{2, i+1, k}+\left(1-\delta_{0, k}\right) p \pi_{2, i+1, k-1,}, i \geq 1, k \geq 0,
\end{gathered}
$$

where $\delta_{i, j}$ is the Kronecker's symbol, and the normalization condition is

$$
\pi_{0,0}+\sum_{i=1}^{+\infty} \sum_{k=1}^{+\infty} \pi_{0, i, k}+\sum_{i=1}^{+\infty} \sum_{k=0}^{+\infty} \pi_{1, i, k}+\sum_{i=1}^{+\infty} \sum_{k=0}^{+\infty} \pi_{2, i, k}=1
$$

In order to solve (1)-(4), we introduce the following generating functions:

$$
\Phi_{0}(x, z)=\sum_{i=1}^{+\infty} \sum_{k=1}^{+\infty} \pi_{0, i, k} x^{i} z^{k}, \Phi_{1}(x, z)=\sum_{i=1}^{+\infty} \sum_{k=0}^{+\infty} \pi_{1, i, k} x^{i} z^{k}, \Phi_{2}(x, z)=\sum_{i=1}^{+\infty} \sum_{k=0}^{+\infty} \pi_{2, i, k} x^{i} z^{k}
$$

and the auxiliary generating functions:

$$
\Phi_{0, i}(z)=\sum_{k=1}^{+\infty} \pi_{0, i, k} z^{k}, \Phi_{1, i}(z)=\sum_{k=0}^{+\infty} \pi_{1, i, k} z^{k}, \Phi_{2, i}(z)=\sum_{k=0}^{+\infty} \pi_{2, i, k} z^{k}, i \geq 1
$$

The following lemma will be used during the derivation of the main result.

Lemma 1: For $0 \leq z<1$, if $\left[\alpha \bar{p} A(\bar{p})+\theta+\bar{\alpha} \theta-\alpha \eta p \beta_{2,1}\right] S(\bar{p}+p \bar{\alpha})-(\bar{p}+p \bar{\alpha})>0$, we have $\Omega(z)>0$, where

$\Omega(z)=S(\tau(z))\left\{\eta[z+(1-z) \bar{p} A(\bar{p})](1-\bar{\alpha} z)(\theta+\theta z) V(\bar{p}+p z)-\alpha \eta z^{2}\right.$

$+(1-z) \bar{\eta}[\bar{p} A(\bar{p})(1-\bar{\alpha} z)(\theta+\theta z)+(\theta+\bar{\alpha} \theta z) z]\}-z(1-z) \tau(z), \tau(z)=\bar{p}+\bar{\alpha} p z$.

Proof: Let us define the following functions

$$
\begin{aligned}
f(z) & =S(\tau(z))\left\{\eta[z+(1-z) \bar{p} A(\bar{p})](1-\bar{\alpha} z)(\theta+\theta z) V(\bar{p}+p z)-\alpha \eta z^{2}\right. \\
& +(1-z) \bar{\eta}[\bar{p} A(\bar{p})(1-\bar{\alpha} z)(\theta+\theta z)+(\theta+\bar{\alpha} \theta z) z]\} \\
g(z) & =z(1-z) \tau(z) .
\end{aligned}
$$


In order to study the slope of the tangent of $f(z)$ and $g(z)$ we calculate:

$f^{\prime}(1)=-\left[\alpha \bar{p} A(\bar{p})+\theta+\bar{\alpha} \theta-\alpha \eta p \beta_{2,1}\right] S(\bar{p}+p \bar{\alpha}), g^{\prime}(1)=-(\bar{p}+p \bar{\alpha})$.

Due to the fact that $f(z)$ and $g(z)$ are convex functions, we observe that if $[\alpha \bar{p} A(\bar{p})+\theta+\bar{\alpha} \theta-$ $\left.\alpha \eta p \beta_{2,1}\right] S(\bar{p}+p \bar{\alpha})-(\bar{p}+p \bar{\alpha})>0$, i.e. $f^{\prime}(1)<g^{\prime}(1)$, then we have $f(z)>g(z)$ in $0 \leq z<1$.

Lemma 2: For $0 \leq z<1$, if $\left[\alpha \bar{p} A(\bar{p})+\theta+\bar{\alpha} \theta-\alpha \eta p \beta_{2,1}\right] S(\bar{p}+p \bar{\alpha})-(\bar{p}+p \bar{\alpha})>0$, the following limits exist

$$
\begin{gathered}
\lim _{z \rightarrow 1} \frac{(1-z) \tau(z)-S(\tau(z))\{(1-z) \eta(\bar{\theta}+\alpha \theta z)+\eta[(1-\alpha z)(\bar{\theta}+\theta z) V(\bar{p}+p z)-\alpha z])}{\Omega(z)} \\
=\frac{\left(\bar{\theta}+\bar{\alpha} \theta-\alpha \eta p \beta_{2,1}\right) S(\bar{p}+p \bar{\alpha})-(\bar{p}+p \bar{\alpha})}{\left[\alpha \bar{p} A(\bar{p})+\bar{\theta}+\bar{\alpha} \theta-\alpha \eta p \beta_{1,1}\right] S(\bar{p}+p \bar{\alpha})-(\bar{p}+p \bar{\alpha})}, \\
\lim _{z \rightarrow 1} \frac{(1-z)(1-\bar{\alpha} z)}{\Omega(z)}=\frac{\alpha}{\left[\alpha \bar{p} A(\bar{p})+\bar{\theta}+\bar{\alpha} \theta-\alpha \eta p \beta_{2,1}\right] S(\bar{p}+p \bar{\alpha})-(\bar{p}+p \bar{\alpha})} .
\end{gathered}
$$

The following theorem gives an explicit expression for the generating function of the stationary distribution of the system state.

Theorem 1: If $\left[\alpha \bar{p} A(\bar{p})+\theta+\bar{\alpha} \theta-\alpha \eta p \beta_{2,1}\right] S(\bar{p}+p \bar{\alpha})-(\bar{p}+p \bar{\alpha})>0$, then

$$
\begin{aligned}
\Phi_{0}(x, z)= & \frac{[A(x)-A(\bar{p})] p x z \pi_{0,0}}{x-\bar{p}} \\
& \cdot \frac{(1-z) \tau(z)-S(\tau(z))\{(1-z) \bar{\eta}(\bar{\theta}+\bar{\alpha} \theta z)+\eta[(1-\bar{\alpha} z)(\bar{\theta}+\theta z) V(\bar{p}+p z)-\alpha z]}{\Omega(z)},
\end{aligned}
$$$$
\Phi_{1}(x, z)=\frac{S(\mathbf{x})-S(\tau(\mathbf{z}))}{\mathrm{x}-\tau(\mathbf{z})} \cdot \frac{(1-z)(1-\bar{\alpha} \mathbf{z}) p x \tau(\mathbf{z}) A(\bar{p}) \pi_{0,0}}{\Omega(\mathbf{z})},
$$$$
\Phi_{2}(x, z)=\frac{V(\mathbf{x})-V(\bar{p}+p z)}{x-(\bar{p}+p z)} \cdot \frac{(1-z)(1-\bar{\alpha} z)(\bar{p}+p z)(\bar{\theta}+\theta z) \eta p x A(\bar{p}) S(\tau(\mathbf{z})) \pi_{0,0}}{\Omega(\mathbf{z})},
$$

where $\pi_{0,0}=\frac{\left[\alpha \bar{p} A(\bar{p})+\bar{\theta}+\bar{\alpha} \theta-\alpha \eta p \beta_{2,1}\right] S(\bar{p}+p \bar{\alpha})-(\bar{p}+p \bar{\alpha})}{\alpha \bar{\theta} A(\bar{p}) S(\bar{p}+p \bar{\alpha})}$. 
Proof: Multiplying (2)-(4) by $z^{k}$ and summing over $k$ and using the boundary condition (1), these equations become

$$
\begin{gathered}
\Phi_{0, i}(\mathrm{z})=\bar{p}\left[\Phi_{0, i+1}(\mathrm{z})+(\bar{\theta}+\theta z) \bar{\eta} a_{i} \Phi_{1,1}(\mathrm{z})+a_{i} \Phi_{2,1}(\mathrm{z})\right]-p a_{i} \pi_{0,0}, i \geq 1, \\
\Phi_{1, i}(\mathrm{z})=\frac{\bar{p}}{z} s_{i} \Phi_{0,1}(\mathrm{z})+\left[\frac{\left(\bar{p} a_{0}+p z\right)(\bar{\theta}+\theta z) \bar{\eta}}{z}-p \alpha z\right] s_{i} \Phi_{1,1}(\mathrm{z})+(\bar{p}+p \bar{\alpha} z) \Phi_{1, i+1}(\mathrm{z}) \\
+p s_{i} \Phi_{0}(1, \mathrm{z})+p \alpha s_{i} z \Phi_{1}(1, \mathrm{z})+\left(p+\frac{\bar{p} a_{0}}{z}\right) s_{i} \Phi_{2,1}(\mathrm{z})+\frac{z-a_{0}}{z} p s_{i} \pi_{0,0}, i \geq 1, \\
\Phi_{2, i}(\mathrm{z})=(\bar{p}+p z)\left[\eta v_{i}(\bar{\theta}+\theta z) \Phi_{1,1}(\mathrm{z})+\Phi_{2, i+1}(\mathrm{z})\right], i \geq 1
\end{gathered}
$$

Multiplying equations (5), (6) by $x_{i}$ and summing over $i$, we get

$$
\begin{gathered}
\left(1-\frac{\bar{p}}{x}\right) \Phi_{0}(\mathrm{x}, \mathrm{z})=\left(A(\mathbf{x})-a_{0}\right)\left[\bar{p} \bar{\eta}(\bar{\theta}+\theta z) \Phi_{1,1}(\mathrm{z})+\bar{p} \Phi_{2,1}(\mathrm{z})-p \pi_{0,0}\right]-\bar{p} \Phi_{0,1}(\mathrm{z}) \\
\left(1-\frac{\tau(\mathrm{z})}{\mathrm{x}}\right) \Phi_{1}(\mathrm{x}, \mathrm{z})=\left[\frac{\bar{\eta}\left(\bar{p} a_{0}+p z\right)(\bar{\theta}+\theta z) S(\mathrm{x})}{z}-\alpha p z S(\mathrm{x})-\tau(\mathrm{z})\right] \Phi_{1,1}(\mathrm{z})+\frac{\left(z-a_{0}\right) p S(\mathrm{x})}{z} \pi_{0,0} \\
+p S(\mathbf{x}) \Phi_{0}(1, z)+\frac{\bar{p} S(\mathbf{x})}{z} \Phi_{0,1}(\mathrm{z})+\alpha p z S(\mathrm{x}) \Phi_{0}(1, z) \Phi_{1}(1, z)+\left(p+\frac{\bar{p} a_{0}}{z}\right) S(\mathbf{x}) \Phi_{2,1}(\mathrm{z}), \\
\left(1-\frac{\bar{p}+p z}{x}\right) \Phi_{2}(\mathrm{x}, \mathrm{z})=(\bar{p}+p z)\left[\eta V(\mathbf{x})(\bar{\theta}+\theta z) \Phi_{1,1}(\mathrm{z})-\Phi_{2,1}(\mathrm{z})\right] .
\end{gathered}
$$

Choosing $x=1$ in (8) and (9), we obtain

$$
\begin{aligned}
& p \Phi_{0}(1, \mathrm{z})=\left(1-a_{0}\right)\left[\bar{p} \bar{\eta}(\bar{\theta}+\theta z) \Phi_{1,1}(\mathrm{z})+\bar{p} \Phi_{2,1}(\mathrm{z})-p \pi_{0,0}\right]-\bar{p} \Phi_{0,1}(\mathrm{z}), \\
& p z(1-\mathrm{z}) \Phi_{1}(1, \mathrm{z})= \bar{p}(1-\mathrm{z}) \Phi_{0,1}(\mathrm{z})+\left\{\bar{\eta}\left[\mathrm{z}+\bar{p} a_{0}(1-\mathrm{z})\right](\bar{\theta}+\theta z)-(\bar{p}+p z) z\right\} \Phi_{1,1}(\mathrm{z}) \\
&+\left[\mathrm{z}+\bar{p} a_{0}(1-\mathrm{z})\right] \Phi_{2,1}(\mathrm{z})-p a_{0}(1-\mathrm{z}) \pi_{0,0} .
\end{aligned}
$$

Substituting above equations(11) and (12) into Equation (9), we get

$$
\begin{gathered}
\left(1-\frac{\tau(\mathrm{z})}{\mathrm{x}}\right) \Phi_{1}(\mathrm{x}, \mathrm{z})=\frac{(1-\bar{\alpha} z) S(\mathrm{x})}{z}\left[\bar{p} \Phi_{0,1}(\mathrm{z})-p a_{0} \pi_{0,0}\right] \\
+\left\{\bar{\eta} S(\mathrm{x})\left[\frac{\left[\mathrm{z}+\bar{p} a_{0}(1-\mathrm{z})\right](\bar{\theta}+\theta z)}{z}+\alpha\left(\bar{p} a_{0}(\bar{\theta}+\theta z)-\theta z\right)\right]-\frac{\alpha \eta z S(\mathrm{x})}{1-z}-\tau(\mathrm{z})\right\} \Phi_{1,1}(\mathrm{z}) \\
+(1-\alpha z) S(\mathrm{x})\left(\frac{1}{1-z}+\frac{\bar{p} a_{0}}{z}\right) \Phi_{2,1}(\mathrm{z})
\end{gathered}
$$


Setting $x=\bar{p}$ in (8), $x=\tau(z)$ in (13) and $x=\bar{p}+p z$ in (10), we obtain

$$
\begin{gathered}
p\left[A(\bar{p})-a_{0}\right] \pi_{0,0}=-\bar{p} \Phi_{0,1}(\mathbf{z})+\left[A(\bar{p})-a_{0}\right]\left[\bar{p} \bar{\eta}(\bar{\theta}+\theta z) \Phi_{1,1}(\mathrm{z})+\bar{p} \Phi_{2,1}(\mathrm{z})\right], \\
\frac{(1-\bar{\alpha} z) S(\tau(\mathbf{z}))}{z} p a_{0} \pi_{0,0}=\frac{(1-\bar{\alpha} z) S(\tau(\mathbf{z}))}{z} \bar{p} \Phi_{0,1}(\mathbf{z}) \\
+\left\{\bar{\eta} S(\tau(\mathbf{z}))\left[\frac{\left.\mathrm{z}+\bar{p} a_{0}(1-\mathrm{z})\right](\bar{\theta}+\theta z)}{z}+\alpha\left(\bar{p} a_{0}(\bar{\theta}+\theta z)-\theta z\right)\right]-\frac{\alpha \eta z S(\tau(\mathbf{z}))}{1-z}-\tau(\mathbf{z})\right\} \Phi_{1,1}(\mathbf{z}) \\
+(1-\bar{\alpha} z) S(\tau(\mathbf{z}))\left(\frac{1}{1-z}+\frac{\bar{p} a_{0}}{z}\right) \Phi_{2,1}(\mathrm{z}) . \\
\Phi_{2,1}(\mathbf{z})=\eta V(\bar{p}+p z)(\bar{\theta}+\theta z) \Phi_{1,1}(\mathbf{z}) .
\end{gathered}
$$

Therefore, from above equations (14)-(16), we find the auxiliary generating functions $\Phi_{0,1}(z)$, $\Phi_{1,1}(\mathrm{z})$ and $\Phi_{2,1}(\mathrm{z})$ as follows:

$$
\begin{gathered}
\Phi_{0,1}(\mathbf{z})=\frac{p z\left[A(\bar{p})-a_{0}\right] \pi_{0,0}}{\bar{p}} \\
. \frac{(1-z) \tau(z)-S(\tau(z))\{(1-z) \eta(\bar{\theta}+\bar{\alpha} \theta z)+\eta[(1-\bar{\alpha} z)(\bar{\theta}+\theta z) V(\bar{p}+p z)-\alpha z]}{\Omega(z)} \\
\Phi_{1,1}(z)=\frac{p A(\bar{p})(1-z)(1-\bar{\alpha} z) S(\tau(\mathbf{z})) \pi_{0,0}}{\Omega(z)}, \\
\Phi_{2,1}(\mathbf{z})=\frac{p A(\bar{p})(1-z)(1-\alpha z) S(\tau(z)) V(\bar{p}+p z)(\bar{\theta}+\theta z) \pi_{0,0}}{\Omega(z)} .
\end{gathered}
$$

Using Lemmas 1 and 2 , the auxiliary generating functions $\Phi_{0,1}(z), \Phi_{1,1}(z)$ and $\Phi_{2,1}(z)$ are well defined for $z \in[0,1)$ and can be extended by continuity for $z=1$ if $\left[\alpha \bar{p} A(\bar{p})+\bar{\theta}+\bar{\alpha} \theta-\alpha \eta p \beta_{2,1}\right] S(\bar{p}+p \bar{\alpha})-(\bar{p}+p \bar{\alpha})>0$.

Substituting (17)-(19) into (8), (10) and (13), we get the generating functions $\Phi_{0}(x, z), \Phi_{1}(x, z)$ and $\Phi_{2}(x, z)$, and in Theorem 1. From the relationship $\pi_{0,0}+\Phi_{0}(1,1)+\Phi_{1}(1,1)+\Phi_{2}(1,1)=1$ we can find the unknown constant $\pi_{0,0}$.

Some marginal generating functions of the number of customers in different conditions are summarized in the following corollary 1 . 


\section{Corollary 1:}

- The marginal generating function of the number of customers in the orbit when the server is idle is given by $\pi_{0,0}+\Phi_{0}(1, z)=\frac{A(\bar{p})\left\{S(\tau(z))\left[M(z)-\alpha \eta z^{2}\right]-(1-z) z \tau(z)\right\} \pi_{0,0}}{\Omega(z)}$, where $M(z)=(1-z) \bar{\eta}[(\bar{\theta}+\bar{\alpha} \theta z) z+\bar{p}(1-z \bar{\alpha})(z \theta+\bar{\theta})]+\eta(\bar{p}+p z)(1-z \bar{\alpha})(\bar{\theta}+\theta z) V(\bar{p}+p z)$.

- The marginal generating function of the number of customers in the orbit when the server is busy is given by $\Phi_{1}(1, z)=\frac{(1-z)[1-S(\tau(z))] \tau(z) A(\bar{p}) \pi_{0,0}}{\Omega(z)}$.

- The marginal generating function of the number of customers in the orbit when the server is on vacation is given by

$$
\Phi_{2}(1, z)=\frac{(1-\bar{\alpha} z)[1-V(\bar{p}-p z)](\bar{p}-p z)(\bar{\theta}+\theta z) \eta A(\bar{p}) S(\tau(z)) \pi_{0,0}}{\Omega(z)} .
$$

- The probability generating function of the orbit size (i.e., of the variable $N$ ) is given by $\Psi(\mathrm{z})=\pi_{0,0}+\Phi_{0}(1, z)+\Phi_{1}(1, z)+\Phi_{2}(1, z)=\frac{F(z) \pi_{0,0}}{\Omega(z)}$ where $F(z)=A(\bar{p})\left\{(1-z)^{2} \tau(z)-[\tau(z)(1-z)+N(z)-\bar{p}(1-z \bar{\alpha})(\eta+(1-z) \bar{\eta})(z \theta+\bar{\theta})] S(\tau(z))\right\}$, $\mathrm{N}(z)=z^{2} \eta(\alpha-p \theta)+z\{z \theta \bar{\alpha}[p z \eta+(-1+z) \bar{\eta}]+[p \eta(-1+z \bar{\alpha})+(-1+z) \bar{\eta}] \bar{\theta}\}$.

- The probability generating function of the system size (i.e., of the variable $L$ ) is given by $\Phi(\mathrm{z})=\pi_{0,0}+\Phi_{0}(1, z)+z \Phi_{1}(1, z)+\Phi_{2}(1, z)=\frac{A(\bar{p}) S(\tau(\mathrm{z}))\left\{\bar{p}(1-z \bar{\alpha})[\eta+(1-z) \bar{\eta}](z \theta+\bar{\theta})-z H(\mathrm{z}) \mid \pi_{0,0}\right.}{\Omega(\mathrm{z})}$, where $H(z)=\tau(z)(1-z)+(\alpha-p \theta) \eta z+z \theta \bar{\alpha}[p z \eta+(1-z) \bar{\eta}]-[p \eta(1-z \bar{\alpha})+(1-z) \bar{\eta}] \bar{\theta}$.

Some performance measures for the system at the stationary regime are summarized in the following corollary 2 .

\section{Corollary 2:}

- The system is free with probability

$$
\pi_{0,0}=\frac{\left[\alpha \bar{p} A(\bar{p})+\bar{\theta}+\bar{\alpha} \theta-\alpha \eta p \beta_{2,1}\right] S(\bar{p}+p \bar{\alpha})-(\bar{p}+p \bar{\alpha})}{\alpha \bar{\theta} A(\bar{p}) S(\bar{p}+p \bar{\alpha})} .
$$

- The system is occupied with probability

$$
\Phi_{0}(1,1)+\Phi_{1}(1,1)+\Phi_{2}(1,1)=\frac{\left[\alpha A(\bar{p})(\bar{\theta}-\bar{p})-\bar{\theta}-\bar{\alpha} \theta+\alpha \eta p \beta_{2,1}\right] S(\bar{p}+p \bar{\alpha})+(\bar{p}+p \bar{\alpha})}{\alpha \bar{\theta} A(\bar{p}) S(\bar{p}+p \bar{\alpha})}
$$


- The server is idle with probability

$$
\pi_{0,0}+\Phi_{0}(1,1)=\frac{A(\bar{p})\left\{S(\bar{p}+p \bar{\alpha})\left(\alpha \bar{p}+\theta \bar{\alpha}+\bar{\theta}-p \alpha \eta \beta_{2,1}\right)-(\bar{p}+p \bar{\alpha})\right\}}{\left[\alpha \bar{p} A(\bar{p})+\bar{\theta}+\bar{\alpha} \theta-\alpha \eta p \beta_{2,1}\right] S(\bar{p}+p \bar{\alpha})-(\bar{p}+p \bar{\alpha})} .
$$

- The server is busy with probability

$$
\Phi_{1}(1,1)=\frac{(\bar{p}+p \bar{\alpha}) A(\bar{p})[1-S(\bar{p}+p \bar{\alpha})]}{\left[\alpha \bar{p} A(\bar{p})+\bar{\theta}+\bar{\alpha} \theta-\alpha \eta p \beta_{2,1}\right] S(\bar{p}+p \bar{\alpha})-(\bar{p}+p \bar{\alpha})}
$$

- The server is on vacation with probability

$$
\Phi_{2}(1,1)=\frac{\alpha p \eta A(\bar{p}) S(\bar{p}+p \alpha) \beta_{2,1}}{\left[\alpha \bar{p} A(\bar{p})+\bar{\theta}+\bar{\alpha} \theta-\alpha \eta p \beta_{2,1}\right] S(\bar{p}+p \bar{\alpha})-(\bar{p}+p \bar{\alpha})} .
$$

- The mean orbit size is given by $E(N)=\Psi^{\prime}(1)=\frac{\pi_{0,0}}{2 \Omega^{\prime}(1)}\left(F^{\prime \prime}(1)\right)-\frac{F^{\prime}(1) \Omega^{\prime \prime}(1)}{\Omega^{\prime}(1)}$.

- The mean system size is given by

$$
E(L)=\Phi^{\prime}(1)=E(N)+\frac{(\bar{p}+p \bar{\alpha}) A(\bar{p})[1-S(\bar{p}+p \bar{\alpha})]}{\left[\alpha \bar{p} A(\bar{p})+\bar{\theta}+\bar{\alpha} \theta-\alpha \eta p \beta_{2,1}\right] S(\bar{p}+p \bar{\alpha})-(\bar{p}+p \bar{\alpha})} .
$$

- The mean time a customer spends in the system (including the service time) is given by

$$
W_{S}=\frac{E(L)}{p} .
$$

\section{Stochastic Decomposition}

A key result in analyzing retrial queues is that the number of customers in the system in steady-state at a random point in time is distributed as the sum of two independent random variables, one of which is the number of customers in the corresponding standard queue (without retrials), the other variable may have different probabilistic interpretations depending on how the vacations are arranged. We present the stochastic decompositions of our queueing system size distribution in two ways as follows:

$$
\begin{gathered}
\Phi(z)=\Pi_{1}(z) \cdot \frac{\pi_{0,0}+\Phi_{0}(1, z)+\Phi_{2}(1, z)}{\pi_{0,0}+\Phi_{0}(1,1)+\Phi_{2}(1,1)}, \\
\Phi(z)=\Pi_{2}(z) \cdot \frac{\pi_{0,0}+\Phi_{0}(1, z)}{\pi_{0,0}+\Phi_{0}(1,1)},
\end{gathered}
$$


where

$$
\begin{aligned}
\Pi_{1}(z)= & \frac{S(\tau(z))[\bar{p}(1-\bar{\alpha} z)(z \theta+\bar{\theta})+(\bar{\theta}+\bar{\alpha} \theta z) z-\tau(z) z]}{S(\tau(z))[\bar{p}(1-\bar{\alpha} z)(z \theta+\bar{\theta})+(\bar{\theta}+\bar{\alpha} \theta z) z]-\tau(z) z} \cdot \frac{[\alpha \bar{p}+\bar{\theta}+\bar{\alpha} \theta] S(\bar{p}+p \bar{\alpha})-(\bar{p}+p \bar{\alpha})}{\alpha \bar{\theta} S(\bar{p}+p \bar{\alpha})}, \\
\Pi_{2}(z)= & \frac{S(\tau(z))\{\bar{p}(1-z \bar{\alpha})[\eta+(1-z) \bar{\eta}](z \theta+\bar{\theta})-z H(z)\}}{\wedge(z)} . \\
& \frac{\left(1-\alpha \theta+\alpha \bar{p}-\alpha \eta p \beta_{2,1}\right) S(\bar{p}+p \bar{\alpha})-(\bar{p}+p \bar{\alpha})}{\alpha \bar{\theta} S(\bar{p}+p \bar{\alpha})}, \\
\wedge(z)= & S(\tau(z))\{(1-z) \bar{\eta}[\bar{p}(1-z \bar{\alpha})(z \theta-\bar{\theta})+z(z \theta \bar{\alpha}+\bar{\theta})] \\
& \left.-z^{2} \alpha \eta+\eta(p z+\bar{p})(1-z \bar{\alpha})(z \theta+\bar{\theta}) V(p z+\bar{p})\right\}-z(1-z) \tau(z) .
\end{aligned}
$$

In the expression (20), the first part is the probability generating function of the customers' number in the standard Geo/G/1 queue with preemptive resume and feedback; the second part is the probability generating function of the blocked customers' number when the server is idle or on vacation. Likewise, the first part in the formula (21) corresponds to the probability generating function of the customers' number in the Geo/G/1 queue with Bernoulli vacation, preemptive resume and feedback; the second part is the probability generating function of the repeated customers when the server is idle. The above results can be summarized in Theorem 2.

\section{Theorem 2:}

- The total customers' number in the queue $L$ can be decomposed as the sum of two independent random variables: $L=L_{1}+M_{1}$, where $L_{1}$ is the customers' number in the standard Geo/G/1 queue with preemptive resume and feedback, and $M_{1}$ is the blocked customers' number when the server is idle or on vacation.

- The total customers' number in the queue $L$ can be decomposed as the sum of two independent random variables: $L=L_{2}+M_{2}$, where $L_{2}$ is the customers' number in the standard queue Geo/G/1 with Bernoulli vacations, preemptive resume and feedback, and $M_{2}$ is the repeated customers' number when the server is idle.

\section{Numerical results}

We will investigate some numerical results of the free probability $\pi_{0,0}$ and the mean time a customer spends in the system $W_{s}$ in relation with some specific para-meters of our model. We assume that the arrival rate $p=0.1$. Furthermore, for the purpose of numerical examples, we suppose that the service time distribution function is geometric with parameter 0.8 , i.e., $S(x)$ $=4 x /(5-x)$, the vacation time distribution function is geometric with parameter 0.6, i.e., $V(x)$ $=3 x /(5-2 x)$, and the retrial times follow a geometric distribution with generating function 
$A(x)=r /[1-(1-r) x]$. We assume that the parametric values are selected under the stable condition in all the below cases.

We have illustrated three curves which correspond to $r=0.15,0.5$ and 0.95 in Figure 2, which describes the behavior of free probability $\pi_{0,0}$ with varying values of $\eta$ by assuming that $\eta=$ $0.5, \theta=0.4$. It is observed in Figure 2 that $\pi_{0,0}$ decreases with increasing the value of $\eta$ and decreases more apparently as the retrial rate $r$ decreases. In Figure 3, we plot the free probability versus $\alpha$ for $\theta=0.4, \eta=0.5$ and three different values of $r$. It is noted that the parameters $\alpha$ does not affect the free probability. In Figure 4 , we plot the free probability $\pi_{0,0}$ versus $\theta$ for $\alpha=0.4, r=0.5$ and three different values of $\eta$. It is obviously that when the parameters $\theta$ and $\eta$ increase, the free probability $\pi_{0,0}$ decreases very rapidly.

Figures 5-7 describe the behavior of the mean time a customer spends in the system with varying values of $\eta, \alpha$ and $\theta$ We give values $\alpha=0.5$ and $\theta=0.4$ in Figure $5 ; r=0.5$ and $\eta=$ 0.4 in Figure 6; $\alpha=0.5$ and $\eta=0.3$ in Figure 7. As it can be seen from Figure 5 and Figure 7, with the increase of parameters $\eta$ and $\theta$, the mean time a customer spends in the system increases. The reason is that increasing vacation probability and feedback to the orbit probability, directly lead to customer's system waiting time longer and more customers in the system. The server becomes busier by increasing the retrial rate $r$ which leads to $W_{s}$ decreases.

The interesting phenomena from Figure 3 and Figure 6 indicate that preemptive parameter $\alpha$ does not affect the free probability $\pi_{0,0}$ and the system waiting time $W_{s}$ is due to the geometric distribution's memory less property.

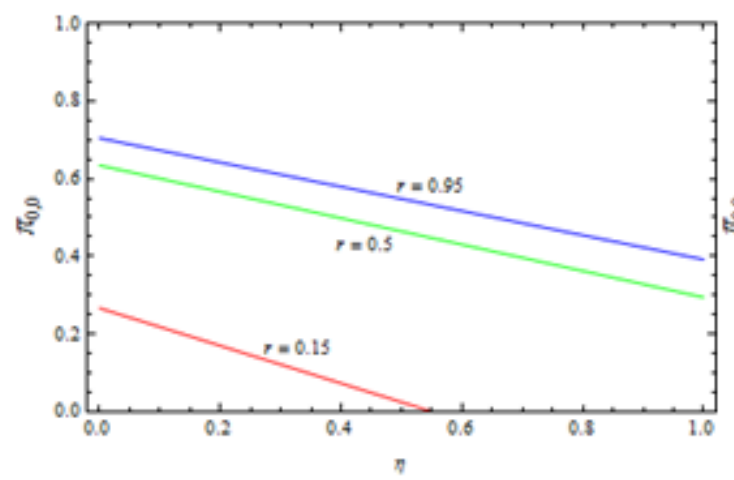

Figure 2. $\pi_{0,0}$ versus $\eta$ for different $r$

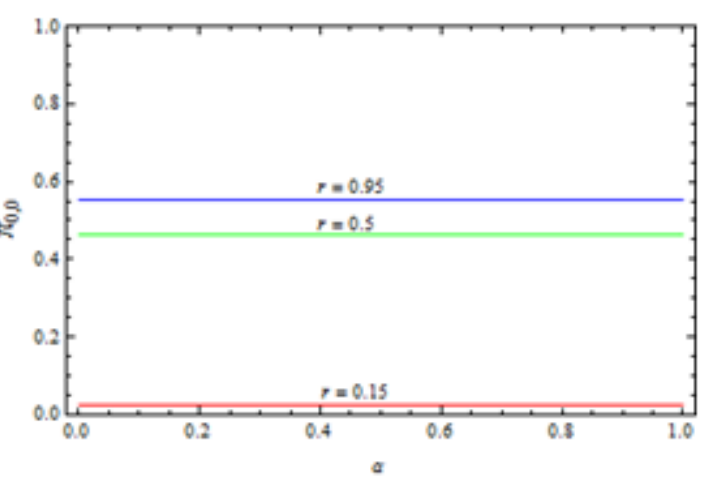

Figure 3. $\pi_{0,0}$ versus $\alpha$ for different $r$ 


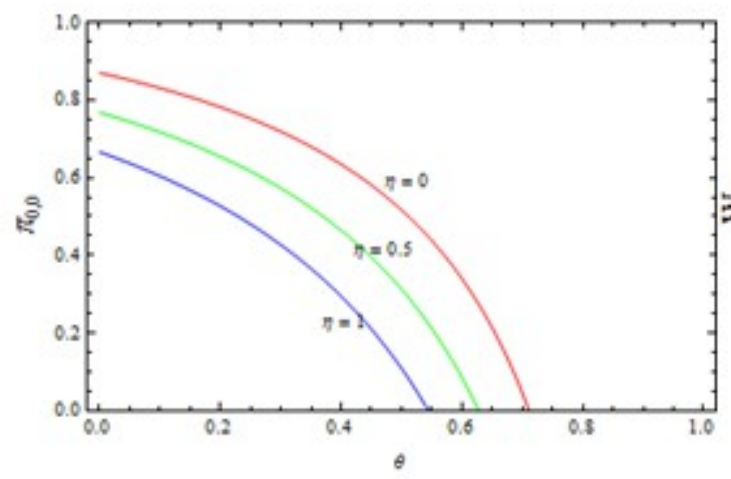

Figure 4. $\pi_{0,0}$ versus $\theta$ for different $\eta$

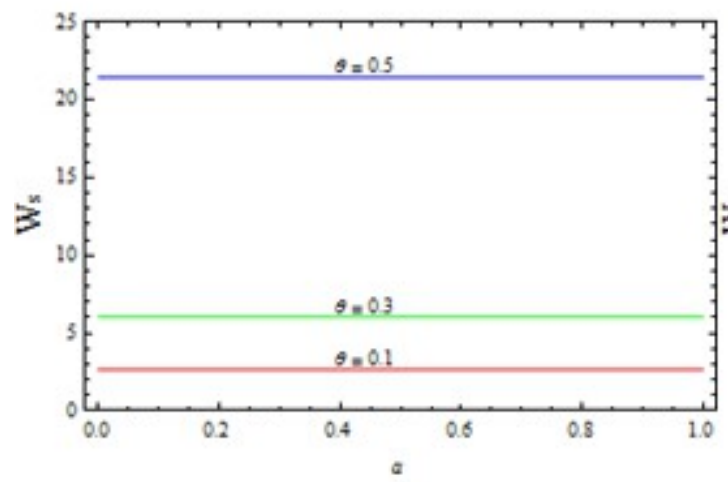

Figure 6. $W_{s}$ versus $\alpha$ for different $\theta$

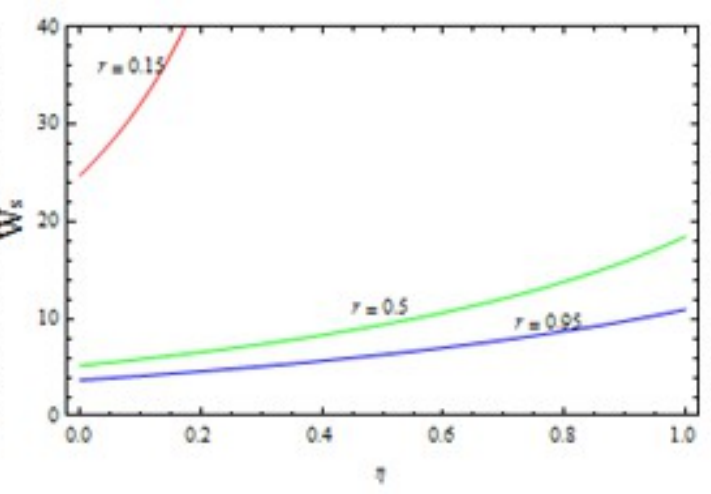

Figure 5. $W_{s}$ versus $\eta$ for different $r$

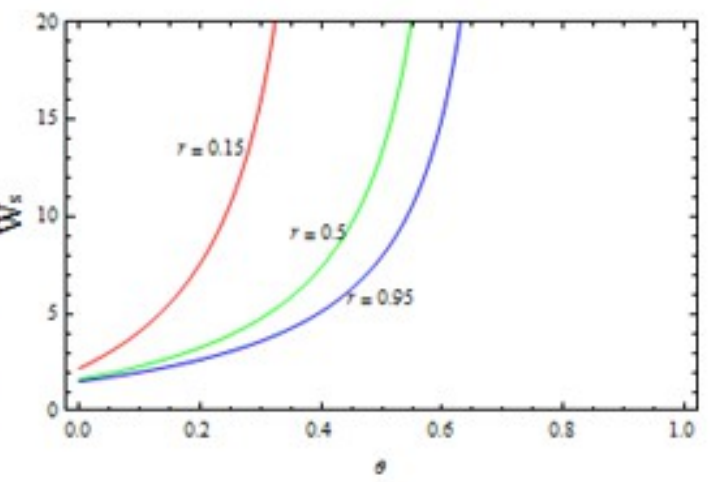

Figure 7. $W_{s}$ versus $\theta$ for different $r$

\section{Conclusions}

In the above analysis, we considered a single server retrial queue with Bernoulli vacation, preemptive resume priority, Bernoulli feedback and general retrial times. It is supposed that the server becomes idle after each service completion and waiting for the next customer with a certain probability, or begins a single vacation process with complementary probability. This model unifies the FCFS and LCFS preemptive resume schedules (for $\alpha=0$ and $\alpha=1$, respectively). The system has been analyzed to obtain the probability generating functions of the system state distribution as well as those of the orbit size and the system size distributions. Hence, we obtain analytical expressions for various performance measures of interest such as idle and busy probabilities, mean orbit and system sizes. Two different stochastic decomposition laws for this system have also been established. Numerical examples have been conducted to investigate the effects of several parameters on the system. 


\section{Acknowledgments}

This research is partially supported by CPSF (2015M572327), NSFC (71131003, 71371075, 71271089), NSFA (KJ2013B162, KJ2015A335, KJ2015A270, KJ2014A174, KJ2013B165), Ministry of Education Foundation of Humanities \& Social Sciences of China (15YJC630053) and Chaohu University Scientific Research Fund. The authors would like to thank the anonymous referees for their valuable comments and suggestions.

\section{References}

Bruneel, H., \& Kim, B.G. (1992). Discrete-time models for communication systems including ATM. Kluwer Academic Publishers.

Choi, B.D., \& Kulkarni, V.G. (1992). Feedback retrial queueing systems. Oxford Statistical Science Series, 93-93.

Kumar, B.K., Vijayakumar, A., \& Arivudainambi, D. (2002). An M/G/1 retrial queueing system with two-phase service and preemptive resume. Annals of Operations Research, 113(1-4), 61-79. http://dx.doi.org/10.1023/A:1020901710087

Kumar, B.K., Vijayalakshmi, G., Krishnamoorthy, A., \& Basha, S.S. (2010). A single server feedback retrial queue with collisions. Computers \& Operations Research, 37(7), 1247-1255. http://dx.doi.org/10.1016/j.cor.2009.04.019

Luo, C., Huang, X., \& Ding, C. (2014). Study on the Departure Process of Discrete-Time Queue with Randomized Vacations. Discrete Dynamics in Nature and Society, Article ID 738021:19. http://dx.doi.org/10.1155/2014/738021

Ramaswamy, R., \& Servi, L.D. (1988). The busy period of the M/G/1 vacation model with a Bernoulli schedule. Communications in Statistics. Stochastic Models, 4(3), 507-521. http://dx.doi.org/10.1080/15326348808807092

Samanta, S.K. (2009). Analysis of a discrete-time GI/Geo/1 queue with single vacation. International Journal of Operational Research, 5(3), 292-310.

http://dx.doi.org/10.1504/IJOR.2009.025198

Servi, L.D. (1986). Average delay approximation of M/G/1 cyclic service queues with Bernoulli schedules. Selected Areas in Communications, IEEE Journal on, 4(6), 813-822. http://dx.doi.org/10.1109/JSAC.1986.1146396

Singh, N., \& Sreenivas, R.S. (2014). Wireless Networks: An Instance of Tandem Discrete-Time Queues. Networks and Communications (NetCom2013), 284, 69-80.

http://dx.doi.org/10.1007/978-3-319-03692-2_6

Takagi, H. (1993). Queueing analysis: A foundation of performance evaluation. Discrete-Time Systems, 3. North-Holland, Amsterdam. 
Wang, J. (2012). Discrete-time Geo/G/1 retrial queues with general retrial time and Bernoulli vacation.Journal of Systems Science and Complexity, 25(3), 504-513. http://dx.doi.org/10.1007/s11424-012-0254-7

Wei, C.M., Qin, Y.Y., \& He, L.X. (2014). A Discrete-Time Geo/G/1 Retrial Queue with Preemptive Resume, Bernoulli Feedback and General Retrial Times. Fuzzy Information \& Engineering and Operations Research \& Management, 211, 539-550. http://dx.doi.org/10.1007/978-3-642-386671_53

Wenhui, Z. (2005). Analysis of a single-server retrial queue with FCFS orbit and Bernoulli vacation. Applied Mathematics and Computation, 161(2), 353-364.

http://doi:10.1016/j.amc.2003.12.032

Wu, J., Liu, Z., \& Peng, Y. (2011). A discrete-time Geo/G/1 retrial queue with preemptive resume and collisions. Applied Mathematical Modelling, 35(2), 837-847. http://dx.doi.org/10.1016/j.apm.2010.07.039

Zhang, Z.G., \& Tian, N. (2001). Discrete time Geo/G/1 queue with multiple adaptive vacations. Queueing Systems, 38(4), 419-429. http://dx.doi.org/ 10.1023/A:1010947911863

Article's contents are provided on an Attribution-Non Commercial 3.0 Creative commons license. Readers are allowed to copy, distribute and communicate article's contents, provided the author's and Journal of Industrial Engineering and Management's names are included. It must not be used for commercial purposes. To see the complete license contents, please visit http://creativecommons.org/licenses/by-nc/3.0/. 